\title{
DIFFUSION COULOMBIENNE MULTIPLE A HAUTE ÉNERGIE DANS L'ÉMULSION NUCLÉAIRE
}

\author{
J. C. MONTRET, B. COUPAT, F. VAZEILlE, L. AVAN \\ Laboratoire de Physique Nucléaire, Faculté des Sciences, Clermont-Ferrand
}

(Reçu le 30 septembre 1969)

\begin{abstract}
Résumé. - L'étude de la diffusion coulombienne multiple à haute énergie et à grandes longueurs de cellule pose le problème de la validité du facteur de diffusion $K$ introduit de manière théorique par Voyvodic et Pickup. Les émulsions nucléaires $K 5$, exposées à des faisceaux de muons de haute énergie $(6 \mathrm{GeV}$ et $12 \mathrm{GeV})$ constituent un matériel de choix pour une telle étude. Nous avons ainsi pu déterminer expérimentalement la valeur de $K$ pour des cellules de $1 \mathrm{~mm}$ à $15 \mathrm{~mm}$ et compléter des résultats encore parcellaires. Dans ce travail, nous étudions également plusieurs méthodes de mesure par diffusion coulombienne multiple.
\end{abstract}

Abstract. - The study of multiple Coulomb scattering for high energies and large cells arises the problem of the validity of the $K$ scattering factor introduced theoretically by Voyvodic and Pickup. $K 5$ nuclear emulsions irradiated in muon beams at 6 and $12 \mathrm{GeV}$ are particularly suited for such a study. We have been able to determine for the first time a complete set of $K$ values for cell length between $1 \mathrm{~mm}$ and $15 \mathrm{~mm}$. Moroever, we present various methods of measurements using the multiple Coulomb scattering technique.

I. INTRODUCTION. - Suivant la technique de Fowler [1], la relation entre le signal de diffusion et le $\mathrm{p} \beta$ d'une particule est :

$$
\frac{p \beta}{z}=\frac{K t^{3 / 2}}{573 \overline{D_{c}}}
$$

$\mathrm{p} \beta / z$ est le moment en $\mathrm{MeV}$ par unité de charge ;

$K$ le facteur de diffusion en

$$
\text { (degré. MeV } \left.(100 \mu \mathrm{m})^{-1 / 2}\right) \text {; }
$$

$t$ la longueur de cellule en $\mu \mathrm{m}$;

$\overline{D_{c}}$ la différence seconde moyenne en valeur absolue due à la diffusion coulombienne multiple en $\mu \mathrm{m}$ pour la cellule $t$.

Pour déterminer le produit $\mathrm{p} \beta$, la connaissance de la variation du facteur de diffusion en fonction de la longueur de cellule $t$ est nécessaire. Les valeurs du facteur de diffusion peuvent être obtenues théoriquement.

Voyvodic et Pickup [2] en utilisant la théorie de Williams [3, 4] ont obtenu une valeur limite de $K$ $(K \simeq 31,3)$ pour les longueurs de cellule supérieures à $1 \mathrm{~cm}$. Ils ont prévu une augmentation possible de $2 \%$ due à la diffusion inélastique par les électrons et une augmentation de $5 \%$ à cause des approximations impliquées dans la loi en $(2 / 3)^{1 / 2}$ permettant de passer de l'angle des tangentes à l'angle des cordes.

D'autres théories : théories de Scott et Snyder [5, 6, $7,8]$, Molière [9], Nigam et col. [10, 11], Cooper et Rainwater [12] permettent d'obtenir les valeurs du facteur de diffusion.
En utilisant des émulsions nucléaires exposées à des particules monocinétiques, de haute énergie, nous nous proposons de comparer certaines méthodes qui utilisent des combinaisons de mesures faites avec des longueurs de cellule différentes et d'étudier la variation du facteur de diffusion en fonction de la longueur de cellule.

II. THÉORIE. - Afin d'utiliser la relation [1] pour déterminer la valeur du facteur de diffusion ou le $\bar{D}_{\mathrm{c}} \mathrm{p} \beta$ de la particule, il faut déterminer la valeur de $D_{\mathrm{c}}$ à partir de la valeur moyenne $\bar{D}$ de la différence seconde mesurée pour la cellule $t$.

Il faut éliminer les contributions des différents bruits qui perturbent les mesures de diffusion.

1. Bruit de platine. - Ce bruit est lié à l'instrument de mesure (bruit mécanique) car le déplacement de la platine n'est pas parfaitement rectiligne.

2. Bruit de grain et de lecture. - Ce bruit est dû à l'imprécision des mesures et à la dispersion des grains le long de la trajectoire de la particule. Il dépend également de la grosseur des grains de l'émulsion.

3. Diffusion parasite. - Lors de son traitement, l'émulsion subit des déformations qui affectent tantôt une grande région de la pellicule comme les courbures, tantôt de petites régions sous forme de dislocations. Cette dernière contribution est désignée sous le nom de diffusion parasite.

II. A. Méthodes utilisant des combinaisons de mesures faites avec des longueurs de cellule différentes. Nous utiliserons les différences secondes quadratiques 
moyennes et non les différences secondes absolues d'où moyennes. Pour une distribution gaussienne :

$$
\left|\overline{D_{\mathrm{c}}}\right|=\sqrt{\frac{2-\overline{D_{\mathrm{c}}^{2}}}{\pi}} \text {. }
$$

Le $\mathrm{p} \beta$ de la particule (de charge unité) est donné par la relation :

$$
\mathrm{p} \beta=\frac{K t^{3 / 2}}{457\left(\overline{D_{c}^{2}}\right)^{1 / 2}} .
$$

Il faut exprimer $\left.\overline{\left(\bar{D}_{\mathrm{c}}^{2}\right.}\right)^{1 / 2}$ et $t$ en $\mu \mathrm{m}, K$ en (degré. $\mathrm{MeV}$ $\left.(100 \mu \mathrm{m})^{-1 / 2}\right)$ pour obtenir $\mathrm{p} \beta$ en $\mathrm{MeV}$.

La différence seconde quadratique moyenne mesurée : $\overline{D^{2}}$ est affectée par un certain nombre d'erreurs (bruit de grain et de lecture, bruit de platine, diffusion parasite). Si $\overline{D_{\mathrm{b}}^{2}}$ est la valeur quadratique moyenne de la différence seconde due à l'ensemble des bruits, on $\mathrm{a}: \overline{D^{2}}=\overline{D_{\mathrm{c}}^{2}}+\overline{D_{\mathrm{b}}^{2}}$. Il existe des méthodes qui permettent, moyennant certaines hypothèses, d'éliminer ces erreurs par combinaisons de mesures faites avec des longueurs de cellule différentes.

Certaines méthodes utilisent les différences troisièmes.

Notations. - Nous désignons respectivement par $\overline{D_{n}^{2}}$ et $\overline{T_{n}^{2}}$ les valeurs quadratiques moyennes des différences secondes et troisièmes mesurées pour une cellule $n t$, par $\overline{D_{\mathrm{c} n}^{2}}$ et $\overline{T_{\mathrm{cn} n}^{2}}$ d'une part, et $\overline{D_{\mathrm{bn} n}^{2}}$ et $\overline{T_{\mathrm{bn}}^{2}}$ d'autre part, les valeurs quadratiques moyennes provenant de la contribution de la diffusion multiple et de l'ensemble des bruits.

$K_{n}$ est le facteur de diffusion multiple pour la cellule $n t$.

A. Hypothèse du bruit constant. - On suppose que la valeur quadratique moyenne de l'ensemble des bruits $\overline{D_{\mathrm{bn}}^{2}}$ est indépendante de la longueur de cellule

$$
\overline{D_{n}^{2}}=\overline{D_{\mathrm{c} n}^{2}}+\overline{D_{\mathrm{b}}^{2}} .
$$

1. Méthode des deux longueurs de cellule [13, 14]. On élimine le terme $\overline{D_{\mathrm{b}}^{2}}$ entre les $\overline{D_{n}^{2}}$ correspondant à deux longueurs de cellule $t$ et $n t$.

$$
\begin{array}{ll}
\text { Pour la cellule } t & \overline{D^{2}}=\overline{D_{\mathrm{c}}^{2}}+\overline{D_{\mathrm{b}}^{2}} \\
\text { Pour la cellule } n t & \overline{D_{n}^{2}}=\overline{D_{\mathrm{c} n}^{2}}+\overline{D_{\mathrm{b}}^{2}} .
\end{array}
$$

Le rapport des différences secondes quadratiques moyennes dues à la diffusion coulombienne pour les cellules $t$ et $n t$ est :

$$
\overline{\overline{D_{\mathrm{c} n}^{2}}}=\left(\frac{K_{n}}{K}\right)^{2} n^{3}
$$

$$
\overline{D_{\mathrm{c}}^{2}}=\frac{\overline{D_{n}^{2}}-\overline{D^{2}}}{n^{3}\left(\frac{K_{n}}{K}\right)^{2}-1} .
$$

a) Méthode "simple ». - En première approximation, on peut considérer $K$ constant sur l'intervalle

$$
t-n t \text {. }
$$

La différence seconde quadratique moyenne due à la diffusion coulombienne multiple pour la cellule $t$ est alors :

$$
\overline{D_{\mathrm{c}}^{2}}=\frac{\overline{D_{n}^{2}}-\overline{D^{2}}}{n^{3}-1} .
$$

La connaissance de $\overline{D_{\mathrm{c}}^{2}}$ permet grâce à la formule (1) d'obtenir le $\mathrm{p} \beta$.

b) Méthode «corrigée ». - En réalité, le facteur de diffusion augmente avec la longueur de cellule, la méthode précédente conduit donc à une surestimation de $\overline{D_{\mathrm{c}}^{2}}$ d'où une sous-estimation de la quantité $\mathrm{p} \beta$.

Pour avoir une meilleure détermination du $\beta$, nous utiliserons les valeurs de $K$ données par la théorie de Voyvodic et Pickup [2], et les formules (4) et (2) conduisent à la quantité $\mathrm{p} \beta$.

Nous avons utilisé ces deux méthodes pour $n=2$ et 4 .

2. Méthode des sommes [13]. - Soient $D_{i}$ et $D_{i+1}$ deux différences secondes successives pour la longueur de cellule $t$

$$
\overline{\left(D_{i}+D_{i+1}\right)^{2}}=\frac{5}{2} \overline{D_{\mathrm{c}}^{2}}+\frac{2}{3} \overline{D_{\mathrm{b}}^{2}} .
$$

En éliminant $\overline{D_{\mathrm{b}}^{2}}$ grâce à la relation (3) :

$$
\overline{D_{\mathrm{c}}^{2}}=\frac{\overline{6\left(D_{i}+D_{i+1}\right)^{2}}-4 \overline{D^{2}}}{11} \text {. }
$$

3. Méthode du centre de gravité [13]. - Les cellules sont groupées $m$ à $m$ et l'on considère le centre de gravité des $m$ cellules :

$$
\overline{y_{i}}=\frac{y_{i}+y_{i+1}+\cdots+y_{i+m-1}}{m} .
$$

On forme les différences secondes sur les $\bar{y}$

$$
\begin{gathered}
D G_{i}(m)=\overline{y_{i}}-2 \overline{y_{i+1}}+\overline{y_{i+2}} \\
\overline{D G^{2}}(m)=\frac{1}{40 m}\left(33 m^{4}+5 m^{2}+2\right) \overline{D_{\mathrm{c}}^{2}}+\frac{\overline{D_{\mathrm{b}}^{2}}}{m} .
\end{gathered}
$$

En éliminant $\overline{D_{\mathrm{b}}^{2}}$ on obtient :

$$
\overline{D_{\mathrm{c}}^{2}}=\frac{40\left(m \overline{D G^{2}}(m)-\overline{D^{2}}\right)}{\left(33 m^{4}+5 m^{2}+2\right)-40} .
$$


Nous appliquons cette formule avec $m=2$ et 4

$$
\mid \begin{array}{ll}
\text { pour } m=2 & \overline{D_{\mathrm{c}}^{2}}=\frac{4\left(2 \overline{D G^{2}}(2)-\overline{D^{2}}\right)}{51} \\
\text { pour } m=4 & \overline{D_{\mathrm{c}}^{2}}=\frac{4\left(4 \overline{D G^{2}}(4)-\overline{D^{2}}\right)}{849}
\end{array}
$$

4. Méthode des sommes et du centre de gravité [9]. Cette méthode est une combinaison des deux précédentes. On forme la somme $D G_{i}(m)+D G_{i+1}(m)$.

$$
\begin{aligned}
\overline{\left(D G_{i}(m)+D G_{i+1}(m)\right)^{2}} & =\frac{1}{60 m} \times \\
& \times\left(138 m^{4}+10 m^{2}+2\right) \overline{D_{\mathbf{c}}^{2}}+\frac{2}{3 m} \overline{D_{\mathbf{b}}^{2}} .
\end{aligned}
$$

Grâce à la relation (2) on obtient :

$$
\overline{D_{\mathrm{c}}^{2}}=\frac{40\left(\frac{3 m}{2} \overline{\left(D G_{i}(m)+D G_{i+1}(m)\right)^{2}}-\overline{D^{2}}\right)}{\left(138 m^{4}+10 m^{2}+2\right)-40} .
$$

Nous utilisons également cette formule avec $m=2$ et $m=4$

$$
\mid \begin{aligned}
\text { pour } m & =2 \\
\overline{D_{\mathrm{c}}^{2}} & =\frac{4\left(3 \overline{\left(D G_{i}(2)+D G_{i+1}(2)\right)^{2}}-\overline{D^{2}}\right)}{221} \\
\text { pour } m & =4 \\
\overline{D_{\mathrm{c}}^{2}} & =\frac{4\left(6 \overline{\left(D G_{i}(4)+\overline{\left.D G_{i+1}(4)\right)^{2}}-\overline{D^{2}}\right)}\right.}{3545}
\end{aligned}
$$

5. Méthode des différences troisièmes [13]. - Nous admettrons la relation :

$$
\overline{T_{n}^{2}}=\frac{3}{2} \overline{D_{\mathrm{c} n}^{2}}+\frac{10}{3} \overline{D_{\mathrm{b}}^{2}} .
$$

En éliminant le bruit entre les relations (8) écrites pour la cellule $t$ et la cellule $n t$, on obtient :

$$
\overline{D_{\mathrm{c}}^{2}}=\frac{\overline{T_{n}^{2}}-\overline{T^{2}}}{\frac{3}{2}\left(n^{3}\left(\frac{K_{n}}{K}\right)^{2}-1\right)} .
$$

Cette formule sera utilisée avec $n=2$.

6. Méthode du rapport constant [14, 15]. - Sans faire d'hypothèse sur la loi de variation des bruits avec la longueur de cellule, on suppose qu'il existe un rapport constant $q^{2}$ entre les différences troisièmes et secondes quadratiques moyennes dues aux bruits.

$$
\overline{T_{\mathrm{bn}}^{2}}=q^{2} \overline{D_{\mathrm{bn}}^{2}}=q^{2} \overline{D_{\mathrm{b}}^{2}} \text {. }
$$

Pour une cellule donnée $n t$

$$
\overline{T_{n}^{2}}=\frac{3}{2} \overline{D_{\mathrm{c} n}^{2}}+q^{2} \overline{D_{\mathrm{b}}^{2}}
$$

et

$$
\overline{D_{n}^{2}}=\overline{D_{\mathrm{cn}}^{2}}+\overline{D_{\mathrm{b}}^{2}}
$$

En éliminant $\overline{D_{\mathrm{b}}^{2}}$ on obtient pour la cellule $t$ :

$$
\overline{D_{\mathrm{c}}^{2}}=\frac{\overline{T^{2}}-q^{2} \overline{D^{2}}}{\frac{3}{2}-q^{2}} \text {. }
$$

En éliminant $\overline{D_{\mathrm{c}}^{2}}$ d'une part entre les relations (10) écrites pour les cellules $t$ et $n t$, et d'autre part entre les relations (2) écrites pour ces mêmes cellules :

$$
q^{2}=\frac{n^{3}\left(\frac{K_{n}}{K}\right)^{2} \overline{T^{2}}-\overline{T_{n}^{2}}}{n^{3} \frac{K_{n}}{K} \overline{D^{2}}-\overline{D_{n}^{2}}} .
$$

\begin{tabular}{|c|c|c|c|c|}
\hline \multicolumn{2}{|l|}{ Méthodes } & $\sqrt{A}$ & $\sqrt{B}$ & $\sqrt{B}$ \\
\hline Sommes & $\ldots \ldots \ldots$ & 1,581 & 0,817 & 1,93 \\
\hline Centre de gravité & $\begin{array}{l}m=2 \\
m=4\end{array}$ & $\begin{array}{l}2,622 \\
7,301\end{array}$ & $\begin{array}{l}0,707 \\
0,500\end{array}$ & $\begin{array}{r}3,70 \\
14,60\end{array}$ \\
\hline $\begin{array}{r}\text { Sommes et centre } \\
\text { de gravité ..... }\end{array}$ & $\begin{array}{l}m=2 \\
m=4\end{array}$ & $\begin{array}{r}4,330 \\
12,120\end{array}$ & $\begin{array}{l}0,577 \\
0,408\end{array}$ & $\begin{array}{r}7,57 \\
29,69\end{array}$ \\
\hline Différences troisièr & & 1,224 & 1,823 & 0,67 \\
\hline
\end{tabular}

Les relations (13), (12) et (2) permettent donc d'obtenir le $\mathrm{p} \beta$ de la particule.

Nous utiliserons $n=2$.

Toutes ces méthodes font intervenir des combinaisons de différences secondes coulombiennes et de différences secondes dues aux bruits.

Ces combinaisons sont du type $\overline{A D_{\mathrm{c}}^{2}}+\overline{B D_{\mathrm{b}}^{2}}$; elles sont caractérisées par les facteurs $\sqrt{A}$ et $\sqrt{B}$ qui multiplient respectivement le signal et le bruit.

Le rapport signal/bruit est donc multiplié par $\sqrt{A / B}$.

Le tableau I comporte les valeurs des facteurs $\sqrt{A}$ et $\sqrt{B}$ ainsi que du rapport $\sqrt{A / B}$ pour les différentes méthodes.

\section{TABLEAU I}

Une méthode sera d'autant plus intéressante que le rapport $\sqrt{A / B}$ sera important, c'est-à-dire si elle diminue le bruit par rapport au signal.

B. HYPOTHÈSE DU BRUIT DE LA FORME $B t^{2 x}$ : MÉTHOde DeS TROIS LONGUEURS DE CELLULE $[14,16]$. Cette méthode a l'avantage de tenir compte de la diffusion parasite. L'étude de la diffusion parasite $[16,17$, $18,19]$ a montré que les différences secondes dues à cette diffusion sont de la forme :

$$
\overline{D_{\mathrm{dp}}}=b t^{x}
$$

$t$ étant la longueur de cellule, $x$ exposant numérique.

La valeur quadratique moyenne sera de la forme

$$
\overline{D_{\mathrm{dp}}^{2}}=B t^{2 x}
$$


donc

$$
\overline{D^{2}}=\overline{D_{\mathrm{c}}^{2}}+B t^{2 x} \text {. }
$$

On utilise trois longueurs de cellule : $t, 2 t, 4 t$.

En éliminant le bruit entre les relations (14) correspondant aux cellules $t$ et $2 t$ on obtient :

$$
\overline{D_{\mathrm{c}}^{2}}=\frac{\overline{D_{2}^{2}}-C \overline{D^{2}}}{8\left(\frac{K_{2}}{K}\right)^{2}-C} .
$$

Dans cette relation, $C$ représente la valeur $2^{2 x}$.

Par élimination de $\overline{D_{\mathrm{c}}^{2}}$ entre les relations (33) correspondant aux cellules $t$ et $2 t$, et aux cellules $2 t$ et $4 t$ on arrive à l'équation :

$$
\frac{\overline{D_{4}^{2}}-8\left(\frac{K_{4}}{K_{2}}\right)^{2} \overline{D_{2}^{2}}}{\left(\frac{K}{K_{2}}\right)^{2} \overline{D_{2}^{2}}-8 \overline{D^{2}}}=\frac{C\left(C-8\left(\frac{K_{4}}{K_{2}}\right)^{2}\right)}{\left(\frac{K}{K_{2}}\right)^{2} C-8}
$$

$C$ est donc obtenu par une équation du second degré.

Cette méthode est voisine de celle utilisée par Yash

Pal et A. K. Ray [20] qui avaient obtenu expérimentalement

$$
\overline{D_{\mathrm{dp}}}=m t+C
$$

avec des cellules $t, 2 t$ et $4 t$ ils ont obtenu :

$$
p=\frac{K t^{3 / 2}}{573}\left(\frac{24}{\bar{D}_{4}^{2}-6 \bar{D}_{2}^{2}+8 \bar{D}^{2}}\right)^{1 / 2}
$$

mais les différences moyennes $\overline{D_{4}}, \overline{D_{2}}, \bar{D}$ utilisées étaient corrigées pour les bruits autres que la diffusion parasite.

II. B. Variation du facteur de diffusion $K$ en fonction de la longueur de cellule. - La détermination $\mathrm{du}$ facteur de diffusion pour de grandes longueurs de cellule (de l'ordre du centimètre) nécessite la mesure précise des bruits qui perturbent les différences secondes.

Les différences secondes dues au signal de diffusion et aux différents bruits sont liées par la formule [21] :

$$
\bar{D}^{2}=\bar{D}_{\mathrm{c}}^{2}+\bar{E}_{\mathrm{gl}}^{2}+\bar{E}_{\mathrm{p}}^{2}+\bar{D}_{\mathrm{dp}}^{2}
$$

$\bar{D}$ : différence seconde moyenne mesurée pour la cellule $t$.

$\overline{D_{\mathrm{c}}}$ : différence seconde moyenne due à la diffusion coulombienne multiple pour la longueur de cellule $t$.

$\overline{E_{\mathrm{g} 1}}$ : erreur sur la différence observée due aux bruits de grain et de lecture.

$\overline{E_{\mathrm{p}}}$ : erreur sur la différence observée due au bruit de platine.

$\overline{D_{\mathrm{dp}}}$ : erreur sur la différence observée due à la diffusion parasite.

1. Détermination du BRUIT DE GRAin et DE LeCTURE. - Nous utiliserons la méthode de Biswas et col. [22]. Chaque mesure est répétée en une position voisine distante de 50 ou $100 \mu \mathrm{m}$ de la précédente. Pour la cellule $t$, nous avons deux séries de différences secondes et le bruit de grain et de lecture est obtenu grâce à la relation :

$$
\overline{\mid{ }^{1} D-} \overline{{ }^{2} D} \mid=\sqrt{2} \overline{E_{\mathrm{gl}}} .
$$

2. Détermination du BRUIT de platine. - Nous utiliserons la méthode de Boggild et Scharff [23].

Lors d'une première mesure, on repère les ordonnées des points de la trace à intervalles égaux, comme pour une mesure de diffusion. L'émulsion est ensuite retournée face pour face et l'on effectue une seconde mesure sur la même trace ; cette mesure s'effectuant à travers la plaque de verre. Les pointés des deux séries doivent correspondre aux mêmes points de la trace et aux mêmes abscisses du déplacement latéral de la platine.

La demi-somme des ordonnées des pointés correspondants des deux mesures donne le profil du déplacement de la platine, et la demi-différence donne la forme de la trace.

Les différences secondes calculées à partir de la demi-somme des ordonnées pour diverses longueurs de cellule donnent le bruit de platine.

3. Détermination DE la Diffusion PARASITE [21]. La diffusion parasite peut être obtenue par des mesures de diffusion relative, effectuées sur deux traces voisines. Pour ces deux traces, le bruit de platine joue de la même façon et de plus on suppose que la diffusion parasite est la même.

$$
\overline{\left|{ }^{1} D-{ }^{2} D\right|}=\overline{D_{\text {rel }}} \text {. }
$$

Les indices 1 et 2 correspondent respectivement à chacune des deux traces.

De plus :

$$
\bar{D}_{\mathrm{rel}}^{2}=2 \bar{D}_{\mathrm{c}}^{2}+2 \bar{E}_{\mathrm{gl}}^{2} .
$$

Si $\overline{E_{\mathrm{g} 1}}$ est connu, nous pouvons obtenir $\bar{D}_{\mathrm{c}}^{2}$ et par conséquent $\overline{D_{\mathrm{dp}}}$ grâce à la relation (17) si $\overline{E_{\mathrm{p}}}$ est connu.

III. MATÉRIEL EXPÉRIMENTAL. - Nous avons réalisé cette étude grâce à des émulsions Ilford $\mathrm{K} 5$ exposées à des faisceaux monoénergétiques de muons de $6 \mathrm{GeV}$ et $12 \mathrm{GeV}[24,25]$. La résolution en moment est $1,5 \%$ pour les deux faisceaux et la contamination en mésons $\pi$ de $10^{-6}$.

Les mesures sont effectuées avec un microscope Leitz, type Olurd équipé d'un objectif à immersion $100 \mathrm{X}$ et des oculaires $25 \mathrm{X}$. La largeur du champ disponible est $60 \mu \mathrm{m}$.

Nous utilisons une cellule de base de $250 \mu \mathrm{m}$.

La longueur de trace mesurée est de $1,15 \mathrm{~m}$ pour les muons de $6 \mathrm{GeV}$ et $1,23 \mathrm{~m}$ pour les muons de $12 \mathrm{GeV}$. 
IV. RÉSULTATS ET DISCUSSION. - A. COMPARAISON DES MÉTHODES UTILISANT DES COMBINAISONS DE MESURES. - Pour chaque trace, nous calculons le $\mathrm{p} \beta$ à l'aide de toutes les méthodes exposées précédemment. Nous effectuons ce calcul pour des longueurs de cellule $t$ de 500, 750 et $1000 \mu \mathrm{m}$.

Nous utilisons une coupure à quatre fois la valeur moyenne pour les différences secondes et troisièmes, et les valeurs du facteur de diffusion utilisées correspondent au cas avec coupure à quatre fois la valeur moyenne. Ces valeurs de $K$ sont données par la théorie de Voyvodic et Pickup [2].

Nous avons vérifié que la somme des différences secondes en valeur algébrique est sensiblement nulle. Pour les traces mesurées nous avons obtenu une valeur moyenne de 0,096 $\mu \mathrm{m}$. Ceci montre que la déformation est peu importante. La figure (1) montre la distribution des différences secondes en valeur absolue et en valeur algébrique.

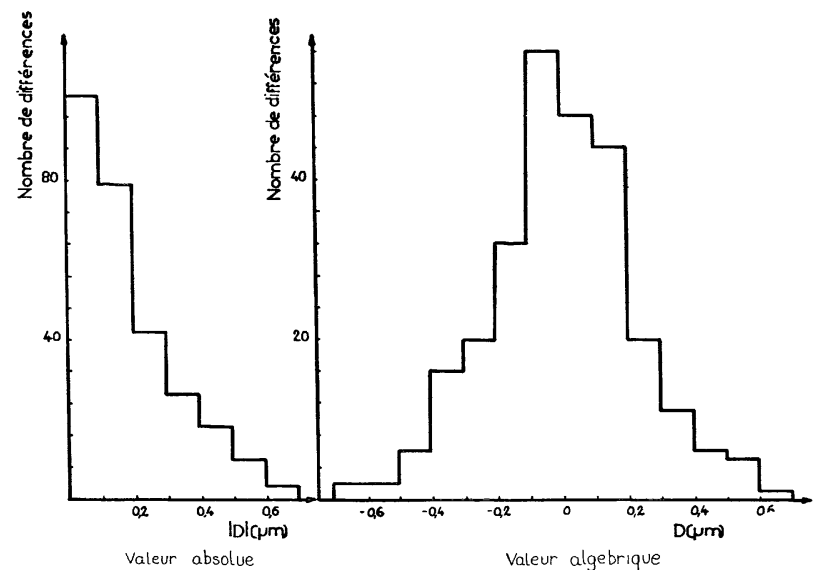

FIG. 1. - Distribution des différences secondes.

Les erreurs sur le produit $\mathrm{p} \beta$ sont obtenues en considérant l'erreur statistique.

Cette erreur est donnée par la formule :

$\Delta(\mathrm{p} \beta) / \mathrm{p} \beta=0,85 / \sqrt{n}$ où $n$ est le nombre de cellules indépendantes. Les valeurs de $\mathrm{p} \beta$ obtenues par les différentes méthodes sont indiquées dans le tableau II.

Ces valeurs correspondent aux moyennes obtenues avec les différentes traces. Pour donner une idée de la

distribution des valeurs de $\mathrm{p} \beta$ obtenues nous avons représenté sur la figure 2 les histogrammes correspondants aux différentes méthodes pour une cellule de $500 \mu \mathrm{m}$.
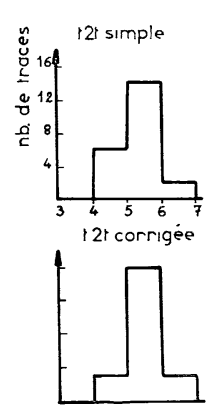

Centre gravité 2

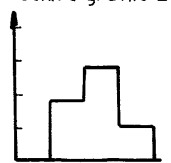

Som cent grav 2

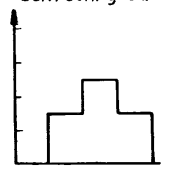

Fig. 2. - Distribution de $\mathrm{p} \beta$ à $t=500 \mu \mathrm{m}$.

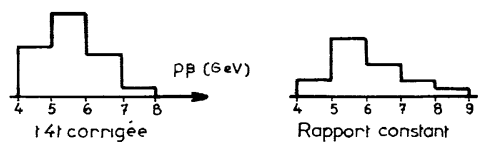

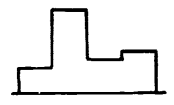

Centre gravite 4

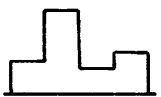

Som.cent grov 4
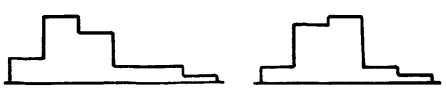

Sommes

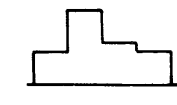

Differences $3^{*}$

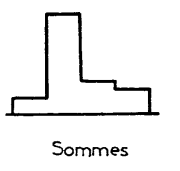

Les résultats sont reportés sur la figure 3 . Cette figure permet de comparer les différentes méthodes. D'une façon générale, l'accord entre les différentes méthodes est bon.
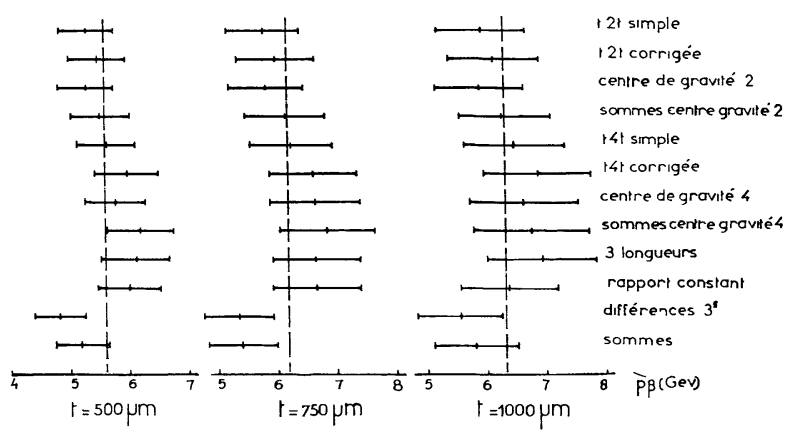

FIG. 3. - Comparaison des différentes méthodes.

\begin{tabular}{|c|c|c|c|c|c|c|c|c|c|c|c|c|c|}
\hline & & & & & & $\mathrm{p} \beta$ en $G$ & & & & & & & \\
\hline $\begin{array}{l}\text { Cellule } \\
(\mu m) \\
-\end{array}$ & $t-2 t$ & $\begin{array}{l}t-2 t \\
\text { corrigé } \\
-\end{array}$ & $\begin{array}{c}\text { Centre } \\
\text { grav. } \\
m=2 \\
-\end{array}$ & $\begin{array}{c}\text { S et } \mathbf{C} \\
\text { grav. } \\
m=2 \\
-\end{array}$ & $\begin{array}{c}t-4 t \\
-\end{array}$ & $\begin{array}{c}t-4 t \\
\text { cor. } \\
-\end{array}$ & $\begin{array}{c}\text { C } \\
\text { grav. } \\
m=4 \\
-\end{array}$ & $\begin{array}{c}S \text { et } C \\
\text { grav. } \\
m=4 \\
-\end{array}$ & 3 long & $\begin{array}{c}\text { Rap. } \\
\text { Cte } \\
-\end{array}$ & $\begin{array}{c}\text { Dif. } \\
3^{\mathrm{e}} \\
-\end{array}$ & Sommes & Moyen. \\
\hline 500 & $\begin{array}{r}5,30 \\
\pm 0,46\end{array}$ & $\begin{array}{r}5,48 \\
\pm 0,48\end{array}$ & $\begin{array}{r}5,28 \\
\pm 0,48\end{array}$ & $\begin{array}{r}5,52 \\
\pm 0,49\end{array}$ & $\begin{array}{r}5,62 \\
+0,50\end{array}$ & $\begin{array}{r}5,96 \\
\pm 0,53\end{array}$ & $\begin{array}{r}5,77 \\
\pm 0,52\end{array}$ & $\begin{array}{r}6,18 \\
\pm 0,57\end{array}$ & $\begin{array}{r}6,12 \\
\pm 0,54\end{array}$ & $\begin{array}{r}5,99 \\
\pm 0,53\end{array}$ & $\begin{array}{r}4,82 \\
\pm 0,42\end{array}$ & $\begin{array}{r}5,19 \\
\pm 0,45\end{array}$ & $\begin{array}{r}5,60 \\
\pm 0,50\end{array}$ \\
\hline 750 & $\begin{array}{r}5,78 \\
\pm 0,62\end{array}$ & $\begin{array}{r}5,99 \\
+0,65\end{array}$ & $\begin{array}{r}5,82 \\
\pm 0,63\end{array}$ & $\begin{array}{r}6,14 \\
\pm 0,68\end{array}$ & $\begin{array}{r}6,23 \\
\pm 0,70\end{array}$ & $\begin{array}{r}6,60 \\
\pm 0,74\end{array}$ & $\begin{array}{r}6,63 \\
\pm 0,76\end{array}$ & $\begin{array}{r}6,84 \\
\pm 0,81\end{array}$ & $\begin{array}{r}6,65 \\
\pm 0,74\end{array}$ & $\begin{array}{r}6,65 \\
\pm 0,73\end{array}$ & $\begin{array}{r}5,34 \\
+0,59\end{array}$ & $\begin{array}{r}5,40 \\
\pm 0,58\end{array}$ & $\begin{array}{r}6,17 \\
\pm 0,69\end{array}$ \\
\hline 1000 & $\begin{array}{r}5,96 \\
\pm 0,75\end{array}$ & $\begin{array}{r}6,17 \\
\pm 0,77\end{array}$ & $\begin{array}{r}5,90 \\
\pm 0,75\end{array}$ & $\begin{array}{r}6,30 \\
\pm 0,82\end{array}$ & $\begin{array}{r}6,50 \\
\pm 0,85\end{array}$ & $\begin{array}{r}6,88 \\
\pm 0,90\end{array}$ & $\begin{array}{r}6,65 \\
+0,91\end{array}$ & $\begin{array}{r}6,78 \\
\pm 0,97\end{array}$ & $\begin{array}{r}6,95 \\
\pm 0,91\end{array}$ & $\begin{array}{r}6,40 \\
\pm 0,82\end{array}$ & $\begin{array}{r}5,56 \\
\pm 0,71\end{array}$ & $\begin{array}{r}5,83 \\
\pm 0,72\end{array}$ & $\begin{array}{r}6,33 \\
\pm 0,82\end{array}$ \\
\hline
\end{tabular}


La méthode des différences troisièmes conduit cependant dans certains cas à une sous-estimation du $\mathrm{p} \beta$. Cette méthode diminue le rapport signal/bruit (Tableau I), donc l'élimination de la contribution des bruits est moins bonne par rapport aux autres méthodes et ceci conduit à une surestimation de $\overline{D_{c}^{2}}$, donc à une diminution du $\mathrm{p} \beta$.

Les valeurs de $\mathrm{p} \beta$ obtenues augmentent lorsque l'on passe d'une cellule d $500 \mu \mathrm{m}$ à une cellule de $750 \mu \mathrm{m}$ puis $1000 \mu \mathrm{m}$. A $500 \mu \mathrm{m}$ le bruit est important par rapport au signal de diffusion, il est donc difficile de l'éliminer totalement, ce qui donne pour $\mathrm{p} \beta$ une valeur un peu faible.

Lorsque la longueur de cellule augmente le signal de diffusion devient de plus en plus grand par rapport aux bruits et les valeurs de $\mathrm{p} \beta$ augmentent légèrement.

- La méthode du rapport constant fournit la valeur de $q^{2}$, qui est le rapport des différences troisièmes coulombiennes aux différences troisièmes dues aux bruits :

$$
q^{2}=\frac{\overline{T_{\mathrm{b}}^{2}}}{\overline{D_{\mathrm{b}}^{2}}} .
$$

La valeur théorique de $q^{2}$ est $10 / 3$.

Avec une cellule de $500 \mu \mathrm{m}$, nous avons obtenu pour $q^{2}$ la valeur 3,4275 ce qui est très proche de la valeur théorique ; on a $\Delta q^{2} / q^{2}=2 \%$.

- La méthode des trois longueurs fournit une indication sur la variation du bruit en fonction de la longueur de cellule.

Pour une cellule de $500 \mu \mathrm{m}$, la valeur moyenne de $C=2^{x}$ est 1,959 ce qui conduit à $2 \times \sim 0,9$.

Le signal mesuré est donc de la forme :

$$
\overline{D^{2}}=\overline{D_{\mathrm{c}}^{2}}+B t^{0,9} \text {. }
$$

Pour une cellule de $750 \mu \mathrm{m}$ nous obtenons :

$$
\overline{D^{2}}=\overline{D_{\mathrm{c}}^{2}}+B t^{1,3} \text {. }
$$

B. VARIATION DU FACTEUR DE DIFFUSION $K$ EN FONCTION DE LA LONGueur DE CEllule. - 1. Détermination du bruit de grain et de lecture. - Les valeurs du bruit de grain et de lecture sont reportées dans le tableau III pour les muons de 6 et $12 \mathrm{GeV}$.

Sur l'intervalle $0-15 \mathrm{~mm}$, le bruit de grain et de lecture satisfait aux relations :

$$
\begin{aligned}
& \text { à } 6 \mathrm{GeV}: \overline{E_{\mathrm{g} 1}}(\mu \mathrm{m})=0,169+2,52 \times 10^{-3} \mathrm{t}(\mathrm{mm}) \\
& \text { à } 12 \mathrm{GeV}: \overline{E_{\mathrm{gl}}}(\mu \mathrm{m})=0,144+1,3 \times 10^{-3} t(\mathrm{~mm})
\end{aligned}
$$

2. Détermination du bruit de platine. - Nous avons utilisé la méthode de Boggild et Scharff [23].

La figure 4 représente le profil du déplacement de la platine obtenu par deux mesures différentes.

Les valeurs du bruit de platine pour une cellule variant de $250 \mu \mathrm{m}$ à $1,5 \mathrm{~cm}$ sont reportées dans le tableau IV et ces résultats sont représentés sur la figure 5 .
TABLEAU III

\begin{tabular}{ccc}
$t(\mathrm{~mm})$ & $\overline{E_{\mathrm{gl}}} 6 \mathrm{GeV}$ & $\overline{E_{\mathrm{gl}}} 12 \mathrm{GeV}$ \\
\hline- & - & - \\
0,5 & $0,150 \pm 0,023$ & $0,139 \pm 0,021$ \\
1,0 & $0,151 \pm 0,021$ & $0,145 \pm 0,021$ \\
2,0 & $0,162 \pm 0,029$ & $0,145 \pm 0,022$ \\
3,0 & $0,163 \pm 0,015$ & $0,160 \pm 0,015$ \\
4,0 & $0,180 \pm 0,024$ & $0,149 \pm 0,016$ \\
5,0 & $0,197 \pm 0,013$ & $0,159 \pm 0,020$ \\
6,0 & $0,187 \pm 0,018$ & $0,149 \pm 0,021$ \\
7,0 & $0,198 \pm 0,014$ & $0,153 \pm 0,018$ \\
8,0 & $0,190 \pm 0,021$ & $0,155 \pm 0,021$ \\
9,0 & $0,205 \pm 0,018$ & $0,164 \pm 0,017$ \\
10,0 & $0,208 \pm 0,021$ & $0,156 \pm 0,024$ \\
12,5 & $0,215 \pm 0,023$ & $0,160 \pm 0,021$ \\
15,0 & $0,215 \pm 0,010$ & $0,162 \pm 0,024$
\end{tabular}

TABleau IV

$\begin{array}{cc}t(\mathrm{~mm}) & \overline{E_{\mathrm{p}}}(\mu \mathrm{m}) \\ - & - \\ 0,25 & 0,114 \\ 0,50 & 0,132 \\ 0,75 & 0,139 \\ 1,00 & 0,151 \\ 2,00 & 0,157 \\ 3,00 & 0,161 \\ 4,00 & 0,165 \\ 5,00 & 0,178 \\ 6,00 & 0,189 \\ 7,00 & 0,203 \\ 8,00 & 0,227 \\ 9,00 & 0,275 \\ 10,00 & 0,317 \\ 12,50 & 0,427 \\ 15,00 & 0,586\end{array}$

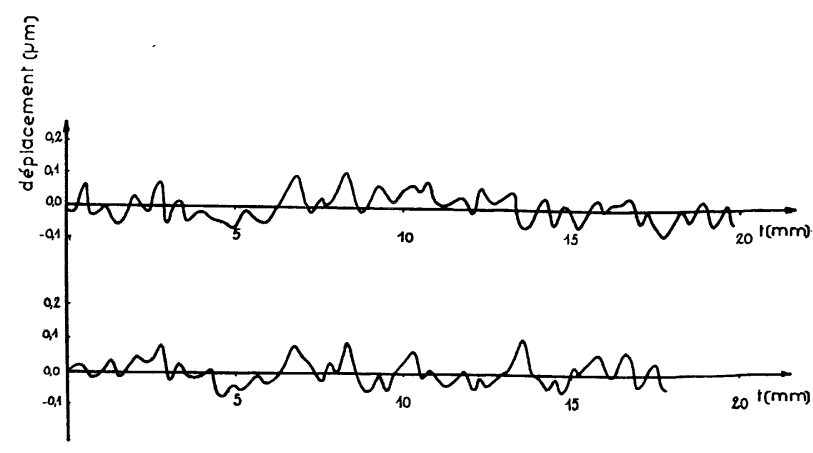

Fig. 4. - Déplacement de la platine.

Au-delà d'un centimètre, la variation devient linéaire et l'on a :

$$
\overline{E_{\mathrm{p}}}(\mu \mathrm{m})=\frac{t(\mathrm{~mm})-4,8}{17,4} .
$$




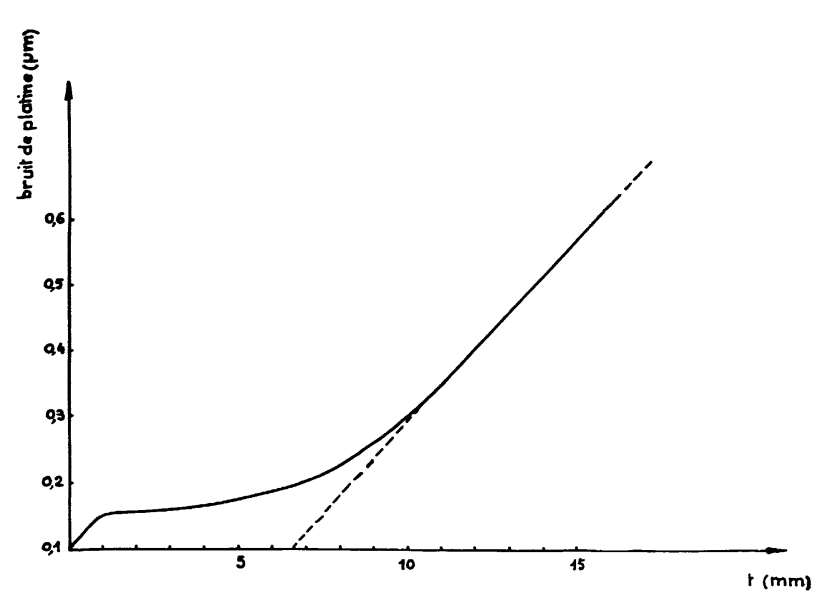

FIG. 5. - Bruit de platine.

3. Valeurs du facteur de diffusion. - Le champ disponible du microscope étant seulement de $60 \mu \mathrm{m}$, il n'a pas été possible d'effectuer suffisamment de mesures de diffusion relative. Nous effectuons les calculs en supposant $\overline{D_{\mathrm{dp}}}=0$.

Nous avons utilisé une coupure à quatre fois la valeur moyenne.

Les résultats à 6 et $12 \mathrm{GeV}$ sont reportés dans le tableau V.
$K=26,78$ ce qui est inférieur à la valeur théorique de Williams. Pour $1 \mathrm{~mm}$ de cellule, il semble donc que la diffusion parasite influe peu. Si la longueur de cellule augmente le signal de diffusion augmente comme $t^{3 / 2}$ et la diffusion parasite comme $t$; l'influence de cette diffusion devient donc de plus en plus faible.

A $12 \mathrm{GeV}$, pour $2 \mathrm{~mm}$ de cellule nous obtenons $K=29,14$, ce qui est légèrement supérieur aux valeurs théoriques. Il semble que l'inflence de la diffusion parasite soit légèrement sensible à cette énergie.

Les valeurs expérimentales sont comparées aux valeurs théoriques sur les figures 6 et 7 . Les valeurs

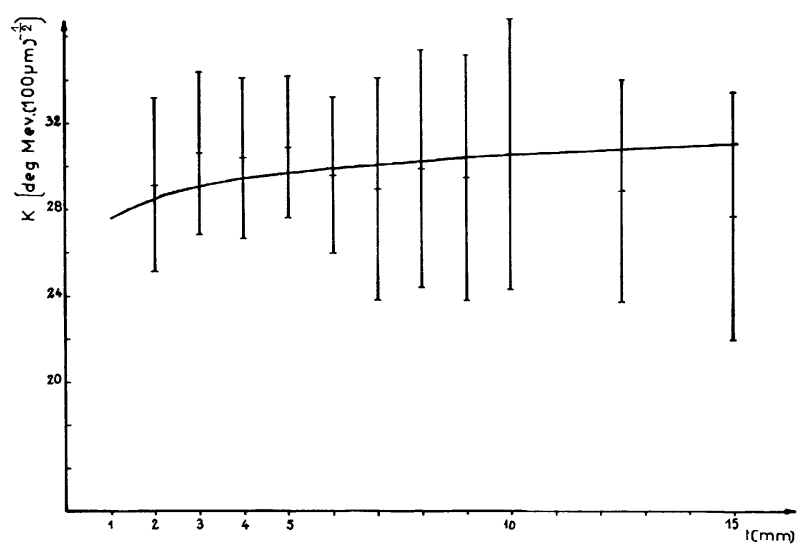

FIG. 6. - Facteur de diffusion à $12 \mathrm{GeV}$.

Tableau V

\begin{tabular}{|c|c|c|c|c|c|c|c|c|c|c|c|c|c|}
\hline $\begin{array}{l}\quad t \mathrm{~mm} \\
\text { Réfé- } \\
\text { rences }\end{array}$ & 1 & 2 & 3 & 4 & 5 & 6 & 7 & 8 & 9 & 10 & 12 & 15 & $\begin{array}{l}\text { Parti- } \\
\text { cule }\end{array}$ \\
\hline [27] & $\begin{array}{r}26,9 \\
\pm 2,4\end{array}$ & $\begin{array}{r}26,5 \\
\pm 3,3\end{array}$ & $\begin{array}{r}25,5 \\
\pm 4,0\end{array}$ & $\begin{array}{r}25,1 \\
\pm 4,6\end{array}$ & & & & $\begin{array}{r}24,7 \\
\pm 5,0\end{array}$ & & & $\begin{array}{r}22,4 \\
\pm 4,5\end{array}$ & & $\begin{array}{c}p \\
5 \text { à } \\
24 \mathrm{GeV}\end{array}$ \\
\hline & $\begin{array}{r}29,4 \\
\pm 2,2\end{array}$ & $\begin{array}{r}25,5 \\
\pm 2,8\end{array}$ & $\begin{array}{r}27,0 \\
\pm 3,6\end{array}$ & $\begin{array}{r}25,2 \\
\pm 4,6\end{array}$ & & & & $\begin{array}{r}25,2 \\
\pm 7,1\end{array}$ & & & $\begin{array}{r}22,7 \\
\pm 6,4\end{array}$ & & $\begin{array}{c}\mu \\
5 \mathrm{GeV}\end{array}$ \\
\hline [28] & & $\begin{array}{c}29,9 \\
\pm 0,92\end{array}$ & & $\begin{array}{r}30,2 \\
\pm 1,5\end{array}$ & & & & $\begin{array}{r}29,0 \\
\pm 2,55\end{array}$ & & $\begin{array}{r}26,9 \\
\pm 1,7\end{array}$ & & & $\begin{array}{c}\pi \\
17 \mathrm{GeV}\end{array}$ \\
\hline & & & & & $\begin{array}{r}27,5 \\
\pm 2,0\end{array}$ & & & & & $\begin{array}{r}27,1 \\
\pm 2,2\end{array}$ & & $\begin{array}{r}26,3 \\
\pm 2,7\end{array}$ & $\begin{array}{c}\pi^{-} \\
17 \mathrm{GeV}\end{array}$ \\
\hline [30] & & & & $\begin{array}{r}32,3 \\
\pm 0,8\end{array}$ & & & & $\begin{array}{r}30,7 \\
\pm 1,0\end{array}$ & & & $\begin{array}{r}30,6 \\
\pm 1,4\end{array}$ & & $\begin{array}{c}\pi^{-} \\
17 \mathrm{GeV}\end{array}$ \\
\hline & & & $\begin{array}{r}30,9 \\
\pm 1,0\end{array}$ & $\begin{array}{r}30,4 \\
+1,1\end{array}$ & $\begin{array}{r}29,0 \\
\pm 1,2\end{array}$ & $\begin{array}{r}28,7 \\
\pm 1,5\end{array}$ & $\begin{array}{r}29,8 \\
\pm 2,2\end{array}$ & $\begin{array}{r}29,2 \\
\pm 2,2\end{array}$ & $\begin{array}{r}31,2 \\
\pm 2,3\end{array}$ & $\begin{array}{r}32,3 \\
\pm 2,4\end{array}$ & & & $\begin{array}{c}\mathbf{P} \\
19,8 \mathrm{GeV}\end{array}$ \\
\hline $\begin{array}{l}\text { Nos } \\
\text { résul- } \\
\text { tats }\end{array}$ & $\begin{array}{r}24,78 \\
\pm 3,02\end{array}$ & $\begin{array}{c}27,84 \\
\pm 3,05 \\
29,14 \\
\pm 4,00\end{array}$ & $\begin{array}{r}26,41 \\
\pm 3,05 \\
30,64 \\
\pm 3,79\end{array}$ & $\begin{array}{r}25,84 \\
\pm 3,75 \\
30,48 \\
\pm 3,74\end{array}$ & $\begin{array}{r}25,53 \\
\pm 3,56 \\
30,88 \\
\pm 3,51\end{array}$ & $\begin{array}{r}25,07 \\
\pm 3,67 \\
29,56 \\
\pm 3,60\end{array}$ & $\begin{array}{r}25,13 \\
\pm 3,87 \\
28,94 \\
\pm 5,23\end{array}$ & $\begin{array}{r}24,86 \\
\pm 3,60 \\
29,85 \\
\pm 5,53\end{array}$ & $\begin{array}{r}23,98 \\
\pm 4,05 \\
29,44 \\
\pm 5,68\end{array}$ & $\begin{array}{r}24,20 \\
\pm 4,10 \\
30,51 \\
\pm 6,80\end{array}$ & $\begin{array}{c}24,56^{*} \\
\pm 4,07 \\
28,82^{*} \\
\pm 5,20\end{array}$ & $\begin{array}{r}24,56 \\
\pm 4,75 \\
27,66 \\
\pm 5,73\end{array}$ & $\begin{array}{c}\mu \\
6 \mathrm{GeV} \\
\mu \\
12 \mathrm{GeV}\end{array}$ \\
\hline
\end{tabular}

(*) Pour $t=12,5 \mathrm{~mm}$.

On peut penser que négliger la diffusion parasite n'entraîne pas de grandes variations du facteur de diffusion.

A $6 \mathrm{GeV}$ pour $1 \mathrm{~mm}$ de cellule, nous obtenons théoriques sont obtenues d'après la théorie de Voyvodic et Pickup, avec coupure à quatre fois la valeur moyenne et en tenant compte de la diffusion inélastique par les électrons. 


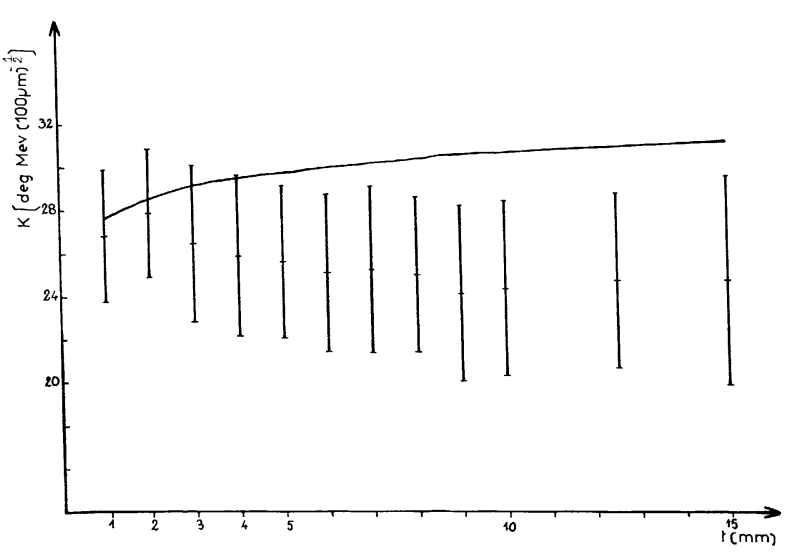

Fig. 7. - Facteur de diffusion à $6 \mathrm{GeV}$.

4. Interprétation des résultats. - Les résultats obtenus montrent une décroissance du facteur de diffusion lorsqu'on augmente la longueur de cellule ce qui est contraire aux lois théoriques. Cet effet est beaucoup plus sensible à $6 \mathrm{GeV}$ qu'à $12 \mathrm{GeV}$.

Cet effet a été signalé par plusieurs auteurs [26, 27, $28,20]$. Le tableau III comporte les valeurs du facteur de diffusion pour des particules de haute énergie obtenues par différents auteurs.

Fichtel et Friedlander [29] ont calculé la variation $\Delta K / K$ qui peut se produire si l'humidité relative $(\mathrm{RH})$ de l'empilement lors de l'exposition est différente du RH standard $(61 \%)$ qui est utilisée pour les calculs théoriques du facteur de diffusion. $\Delta K / K$ varie de $+4 \%$ à $-4 \%$ lorsque $\mathrm{RH}$ varie de $5 \%$ à $87 \%$. La variation d'humidité relative ne permet donc pas d'expliquer la différence entre les valeurs expérimentales et les valeurs théoriques.

P. K. Aditya [30] a expliqué la diminution des valeurs expérimentales du facteur de diffusion aux grandes longueurs de cellule en faisant intervenir des conditions géométriques. La sous-estimation de $K$ est due aux limites imposées lors des mesures au déplacement de la trace de la particule dans l'émulsion.

Soit $f(x, \mathrm{p} \beta \mathrm{c}, d)$ la probabilité pour que la trace d'une particule d'énergie $\mathrm{p} \beta \mathrm{c}(\mathrm{GeV})$ ait un déplacement inférieur à $d(\mu \mathrm{m})$ après avoir parcouru une distance $x(\mathrm{~cm})$ dans l'émulsion.

A partir de mesures sur des muons de $8 \mathrm{GeV}$, Aditya, en imposant différentes limites au déplacement latéral a obtenu :
- pourcentage de sous-estimation $=30(1-f)$ où $f$ désigne la probabilité définie précédemment.

Pour nos mesures le déplacement latéral autorisé était de l'ordre de 50 à $60 \mu \mathrm{m}$ pour un parcours de $5 \mathrm{~cm}$ dans l'émulsion.

Ceci conduit à $f \sim 0,4$ et un pourcentage de sousestimation de $18 \%$ à $6 \mathrm{GeV}$,

— et à $f \sim 0,7$ et un pourcentage de $9 \%$ à $12 \mathrm{GeV}$.

Expérimentalement, nous obtenons :

à $6 \mathrm{GeV}$ : une sous-estimation de $21 \%$ pour des cellules de l'ordre du centimètre ;

à $12 \mathrm{GeV}$ : une sous-estimation de $8 \%$ environ pour ces mêmes cellules.

Ces résultats sont donc en accord avec ceux d'Aditya.

Conclusion. - Les mesures de diffusion coulombienne multiple donnent des résultats corrects dans le domaine des hautes énergies (quelques $\mathrm{GeV}$ ). En particulier, il faut noter la parfaite cohérence des résultats obtenus par les différentes méthodes avec des cellules de longueurs relativement modestes (Fig. 2 et Fig. 3). Cependant, la méthode qui semble la mieux adaptée à des mesures de ce type semble être la méthode « centre de gravité 2 ». En effet, la figure 2 nous montre pour cette méthode une distribution nettement "piquée » et sur la figure 3 la valeur moyenne de cette méthode apparaît concordante parfaitement avec la valeur moyenne générale des douze méthodes utilisées.

A grandes longueurs de cellule, les valeurs du facteur de diffusion coulombienne multiple s'écartent des valeurs théoriques.

Cette diminution qui est très visible sur les figures 3 et 4 semble pouvoir s'expliquer par les conditions géométriques imposées aux mesures.

En effet, nous remarquons l'accord entre le pour centage de sous-estimation déduit des calculs d'Aditya et nos valeurs expérimentales.

Remerciements. - Nous remercions :

- Dr M. L. Perl et W. T. Toner pour leur aide à la préparation de l'expérience et à sa réalisation au Stanford Linear Accelerator Center ;

- Pr. Hofstadter et Dr R. E. Taylor pour l'aide matérielle qu'ils nous ont apportée pendant notre séjour à Stanford.

\section{Bibliographie}

[1] Fowler (P. H.), Phil. Magn., 1950, 41, 169.

[2] Voyvodic (L.) et Pickup (E.), Phys. Rev., 1952, 85, 91.

[3] Williams (E. J.), Proc. Roy. Soc. (London), 1939, 169A, 531 .

[4] Williams (E. J.), Phys. Rev., 1940, 58, 292.

[5] Scott (W. T.), Phys. Rev., 1949, 75, 1753.

[6] Scotr (W. T.), Phys. Rev., 1949, 76, 212.
[7] Scott (W. T.) et SNyder (H. S.), Phys. Rev., 1950, $78,223$.

[8] SNYder (H. S.), Phys. Rev., 1951, 83, 1066.

[9] Molière (G.), Z. Naturf., 1948, 3A, 78.

[10] Nigam (B. P.), Sundaresan (M. K.) et Wu Ta-You, Phys. Rev., 1959, 115, 491.

[11] Nigam (B. P.), Phys. Rev., 1963, 131, 238.

[12] Cooper (L. N.) et Rainwater (J.), J. Phys. Rev., $1955,97,492$. 
[13] Tsai CHU, Technique des mesures dans l'émulsion nucléaire, Clermont-Fd, 1964.

[14] Bermond (J.), Brou (R.), Patou (C.), Korpuskular Photographie IV, 480, München, 1963.

[15] Patou (C.), Thèse de Doctorat d'Etat, Caen, octobre 1966.

[16] Apostolakis (A. J.), Clarke (O. J.) et Major (J. V.), Il Nuovo Cimento, 1957, 5, 337.

[17] Brisbout (F. A.), Danayke (C.), Engler (A.), Fowler (P. H.), Jones (P. B.), Il Nuovo Cimento 1956, 3, 1400.

[18] Lord (J. J.), Chang (D. B.) et Fischer (F. W.), Bul. Am. Phys. Soc., 1956, 1, 250.

[19] JudeX (B.), Il Nuovo Cimento, 1960, 16, 834.

[20] Yash Pal, Ray (A. K.), Il Nuovo Cimento, 1963, 27, 960 .

[21] Dhal Jensen (E.), Gailloud (M.), Lock (W. O.) Vanderhague (G.), Il Nuovo Cimento, 1961, 22,850 .
[22] Biswas (S.), Peters (B.) et Rama, Proc. Ind. Acad Sci., 1955, 41, 154.

[23] Boggild (J. K.), Scharff (M.), Suppl. Nuovo Cimento, 1954, 12, 374.

[24] Montret (J. C.), Rapport Interne au Laboratoire de Physique Nucléaire de Clermont-Ferrand, P. N. C. F., 68 RI.

[25] Cox (J.), Martin (F.), Perl (M. L.), Tan (T. H.), TONER (W. T.), ZIPF (T. F.), LAKIN (W. L.), Slac, PUB 434, Stanford (1968).

[26] Wong (E.) et JaIn (P. L.), Il Nuovo Cimento, 1968, $56,922$.

[27] Hossain (A.), Votruba (M. F.), Wataghin (A.), Evans (D.), Il Nuovo Cimento, 1961, 22, 861.

[28] Bozoki (G.), Gombosi (E.), Jenik (L.), NaGy (E.), SAHINI (M.), $V^{\text {th }}$ International Conference on. Nuclear Photography, IX, 32, Geneva (1964).

[29] Fichtel (C.) et Friedlander (M. W.), Il Nuovo Cimento, 1958, 10, 1032

[30] Aditya (P. K.), Ind. Jour. Phys., 1968, 40, 238. 